\title{
Reduced adhesion of sparkling water droplets
}

\author{
Divya Panchanathan $\odot^{\circ},{ }^{*}$ Philippe Bourrianne, ${ }^{*}$ Kripa K. Varanasi, ${ }^{\dagger}$ and Gareth H. McKinley ${ }^{\dagger}$ \\ Department of Mechanical Engineering, MIT, 77 Massachusetts Avenue, Cambridge, \\ Massachusetts 02119, USA
}

(Received 22 August 2019; published 24 October 2019)

\begin{abstract}
This paper is associated with a video winner of a 2018 APS/DFD Gallery of Fluid Motion Award for work presented at the DFD Gallery of Fluid Motion. The original video is available online at the Gallery of Fluid Motion, https://doi.org/10.1103/APS.DFD.2018. GFM.V0097
\end{abstract}

DOI: 10.1103/PhysRevFluids.4.100505

Liquids are defined by their ability to flow freely when subjected to a shear stress. However, at millimetric scales, water drops eventually adhere to their solid substrate and develop a remarkable ability to resist gravity; common examples include raindrops stuck on windshields, eyeglasses, or other ordinary substrates. Droplet adhesion occurs when contact line pinning arrests the motion of a three-phase contact line and surface tension at the liquid-solid interface overcomes the weight of a liquid droplet [1]. Many strategies have been developed to avoid this sticking behavior by reducing the contact area between a liquid and the underlying substrate. Thus, water-repellent solids are obtained by combining hydrophobic chemistry and submicronic roughness, as predicted by Cassie and Baxter [2] and exhibited by lotus leaves [3]. The liquid then only sits on the tops of the micrometric textures in a fakir state, which results in a drastic reduction of contact area and thus a low-adhesion regime [4]. Here we study the wetting and motion of a carbonated water drop placed on a superhydrophobic coating.

By imaging with an interferometric setup the base (of size $r_{\mathrm{c}}$ ) of a sparkling water drop (i.e., a droplet saturated with dissolved $\mathrm{CO}_{2}$ ) immediately after its deposition on a superhydrophobic coating, we observe a striking delay in the time to wetting. In Fig. 1(a) we show a composite image of the basal area of a carbonated water drop obtained by combining four quadrants imaged at four different times after drop deposition. As time evolves clockwise, the transition to the wetting regime is dramatically modified. Initially, localized pinning points are formed at the drop perimeter (top-right quadrant, as seen at 1:27 in the GFM video); the region of liquid-solid contact then spreads towards the base center (bottom-right quadrant, 1:38 in the video) until eventually covering the entire area (bottom-left, 1:42 in the video). In Fig. 1(b) we present a kymograph (or spatiotemporal diagram) illustrating these dynamics. It is constructed by assembling a grayscale composite image of the diameter $\left(-r_{\mathrm{c}}<r<r_{\mathrm{c}}\right)$ of the droplet base as a function of the time $t$ after deposition. The distribution of light intensity along the diameter continuously varies until reaching a terminal state after $\sim 70 \mathrm{~s}$. Liquid-solid contact points are first restricted to the drop edges (white dashed lines, at $r= \pm r_{\mathrm{c}}$ ) as the rest of the area is covered by a central bubble [Fig. 1(c)]. From

\footnotetext{
*These authors contributed equally to this work.

†Corresponding authors: kripa@mit.edu; gareth@mit.edu
}

Published by the American Physical Society under the terms of the Creative Commons Attribution 4.0 International license. Further distribution of this work must maintain attribution to the author(s) and the published article's title, journal citation, and DOI. 

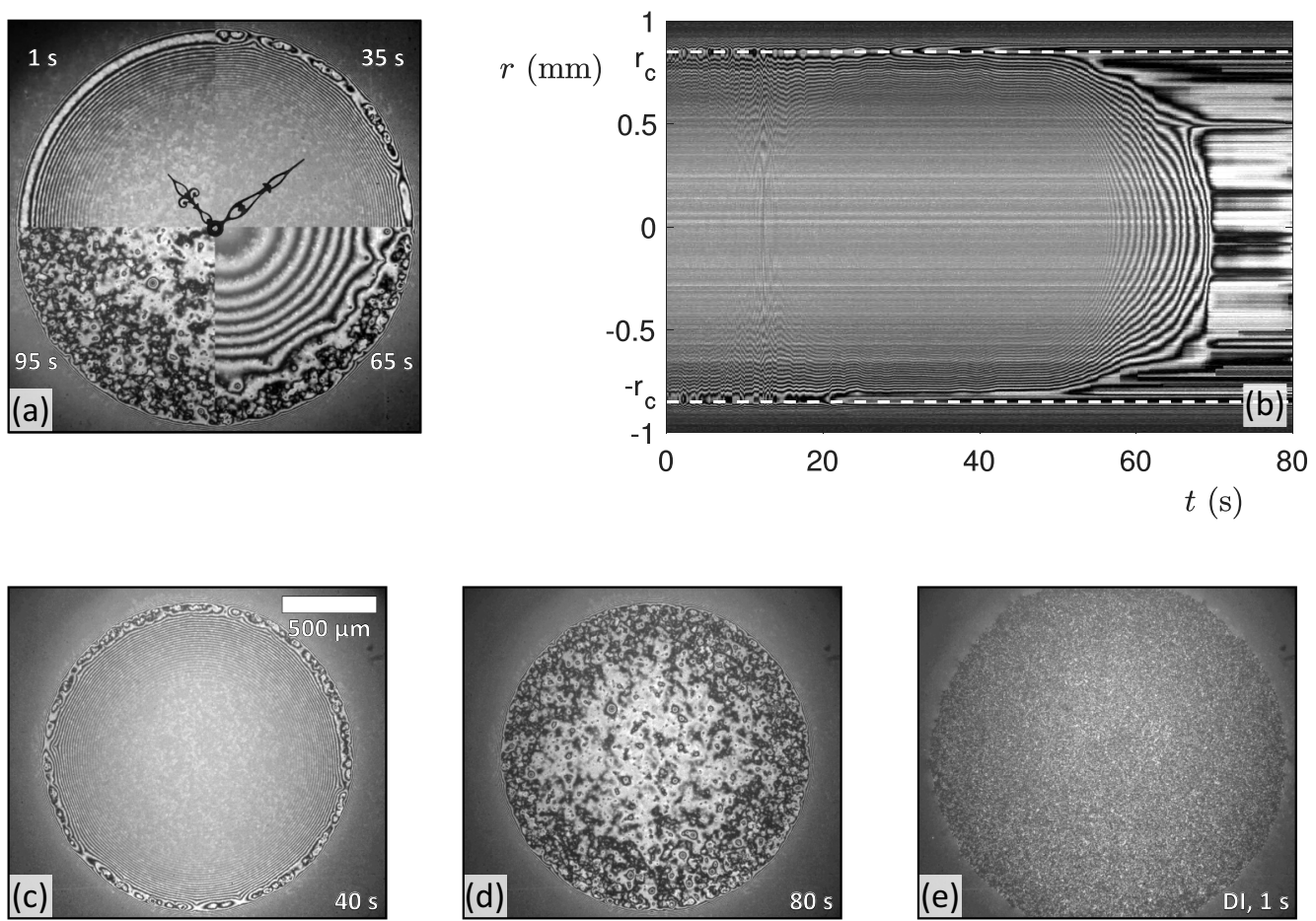

FIG. 1. Wetting regimes on superhydrophobic substrates. (a) Composite image illustrating the wetting evolution of carbonated water drop with time. From the top-left quadrant ( $1 \mathrm{~s}$ after deposition) to the bottom left quadrant (95 s), the topography of the drop base and the associated wetting regime evolves with time (clockwise). (b) Kymograph of the wetting of a carbonated water drop deposited on a superhydrophobic substrate. Contact points are initially localized at the edges $\left(r= \pm r_{\mathrm{c}}\right)$ and spread radially inward until they finally cover the whole area after $70 \mathrm{~s}$. (c) Photography of the base of a soda water drop $40 \mathrm{~s}$ after deposition on the superhydrophobic substrate. (d) Picture of the base of a carbonated water drop $80 \mathrm{~s}$ after deposition. (e) Photography of a DI water drop $1 \mathrm{~s}$ after deposition on the superhydrophobic substrate.

$t \approx 40$ to $70 \mathrm{~s}$, the liquid spreads to the center of the drop base as seen in the kymograph. The wetting has been delayed for several tens of seconds due to the release of $\mathrm{CO}_{2(\mathrm{~g})}$ from the fizzy droplet.

After $70 \mathrm{~s}$, the cessation of dynamical evolution in the interferogram indicates a regular Cassie state as seen in Fig. 1(d) $(t=80 \mathrm{~s})$. The whole contact area is then covered by a myriad of bright and dark patches, which highlights the heterogeneous nature of the wetting in the Cassie-Baxter regime. Similar observations are noticed immediately after the deposition of a deionized (DI) water drop on the same superhydrophobic coating [Fig. 1(e)]. However, the bright and dark spots are markedly larger underneath a soda water drop due to the residual presence of bubbles of $\mathrm{CO}_{2(\mathrm{~g})}$ trapped inside the porosity of the superhydrophobic coating. Liquid-solid contacts are indeed still present, but they are substantially reduced underneath carbonated water drops.

The delay in wetting and the substantial decrease of liquid-solid contacts cause a reduction of the drop adhesion. When deposited on slightly tilted (slope $\alpha \approx 0.3^{\circ}$ ) superhydrophobic solids, water drops (of volume $\Omega \approx 80 \mu \mathrm{l}$ ) slide down the surface due to gravity [Fig. 2(a)]. However, the presence of a wetting defect induces their arrest and capture as seen on the sequence of top-view images shown in Fig. 2(b) (with a timestep of $333 \mathrm{~ms}$ ). A drop of DI water (in blue) is released from a pipette tip on a tilted superhydrophobic solid (top frame). The liquid globule slides down 

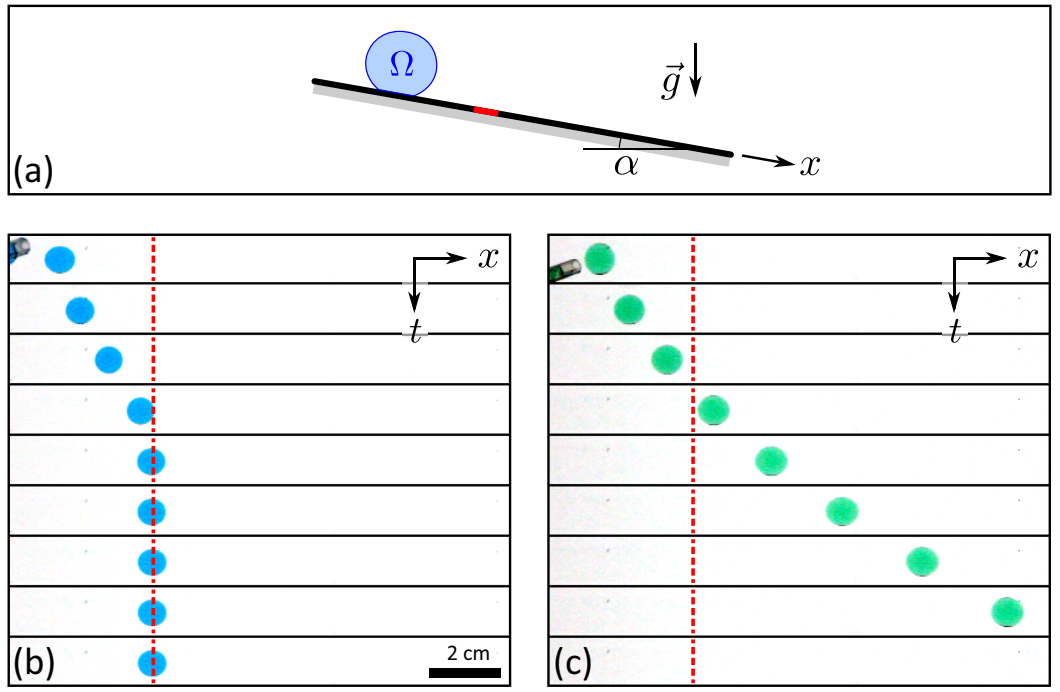

FIG. 2. Carbonated water drops overcome wetting defects that induce contact line pinning. (a) Sketch of the experiment. A drop of volume $\Omega$ is placed on a superhydrophobic substrate tilted by an angle $\alpha \approx 0.3^{\circ}$. A defect (in red) is present on the surface. (b) Sequence of images (separated by timesteps of $333 \mathrm{~ms}$ ) of a DI water (in blue) sliding down the tilted solid until it is arrested by the defect (in red). (c) Sequence of images (timestep of $333 \mathrm{~ms}$ ) of a carbonated water deposited on the solid. The drop overcomes the defect as it accelerates along the inclined plate, translating the entire length of the water-repellent solid.

the slope (from left to right) until it encounters a coating defect (highlighted in red) on which it remains stuck. Conversely, a carbonated water drop [Fig. 2(c), in green] overcomes the surface defect and maintains its motion down the inclined plane, continuing to accelerate along the entire path. Due to the reduction of interfacial adhesion, soda water drops are much less sensitive to surface defects and imperfections, an observation of possible interest to design new antiadhesive technologies.

[1] C. G. L. Furmidge, Studies at phase interfaces. I. The sliding of liquid drops on solid surfaces and a theory for spray retention, J. Colloid Sci. 17, 309 (1962).

[2] A. B. D. Cassie and S. Baxter, Wettability of porous surfaces, Trans. Faraday Soc. 40, 546 (1944).

[3] W. Barthlott and C. Neinhuis, Purity of the sacred lotus, or escape from contamination in biological surfaces, Planta 202, 1 (1997).

[4] A. Lafuma and D. Quéré, Superhydrophobic states, Nat. Mater. 2, 457 (2003). 